Canadian University Music Review

Canadian University Music Review

Revue de musique des universités canadiennes

\title{
La semaine annuelle de musique de Montréal sous les auspices du Delphic Study Club, 1923-1937
}

\section{Marie-Thérèse Lefebvre}

Volume 21, numéro 2, 2001

URI : https://id.erudit.org/iderudit/1014485ar

DOI : https://doi.org/10.7202/1014485ar

Aller au sommaire du numéro

\section{Éditeur(s)}

Canadian University Music Society / Société de musique des universités canadiennes

ISSN

0710-0353 (imprimé)

2291-2436 (numérique)

Découvrir la revue

Citer cet article

Lefebvre, M.-T. (2001). La semaine annuelle de musique de Montréal sous les auspices du Delphic Study Club, 1923-1937. Canadian University Music Review / Revue de musique des universités canadiennes, 21(2), 60-76.

https://doi.org/10.7202/1014485ar

\section{Résumé de l'article}

L'étude présente d'abord le contexte dans lequel fut créé la Semaine de musique annuelle (1923-1937) à Montréal et propose une analyse du mode de fonctionnement, inspiré du modèle américain créé par Charles Milton Tremaine en 1920. L'auteure commente les données sur les collaborateurs, les commanditaires, les bourses, les lieux de concerts, les interprètes, le répertoire et les conférenciers, et fait ressortir les principaux éléments de la critique de ces événements. La conclusion met en évidence la dualité culturelle anglophone-francophone dans la conception de la musique et son rôle dans l'éducation, et souligne l'action des femmes dans la gestion d'organismes de diffusion de la musique au Québec.
All Rights Reserved @ Canadian University Music Society / Société de musique des universités canadiennes, 2002
Ce document est protégé par la loi sur le droit d'auteur. L’utilisation des services d'Érudit (y compris la reproduction) est assujettie à sa politique d'utilisation que vous pouvez consulter en ligne.

https://apropos.erudit.org/fr/usagers/politique-dutilisation/ 


\section{LA SEMAINE ANNUELLE DE MUSIQUE DE MONTRÉAL SOUS LES AUSPICES DU DELPHIC STUDY CLUB, 1923-1937}

\section{Marie-Thérèse Lefebvre}

\section{INTRODUCTION}

Nous nous intéressons depuis plusieurs années aux discours idéologiques qui ont servi de cadre de référence aux activités des compositeurs, critiques, fondateurs et gestionnaires d'organismes musicaux à travers l'histoire musicale du Québec. Quelles conceptions de la musique défendent ces individus? Dans quel contexte social, politique ou économique se développent-elles? À quels courants de pensée peut-on relier les débats esthétiques que ces conceptions, souvent divergentes, ont suscités? Ces interrogations nous ont menée à publier les écrits de certains compositeurs, tels ceux de Serge Garant, Gilles Tremblay, Jean Vallerand et Rodolphe Mathieu. Nous avons par la suite exploré quelques pistes autour de la création des femmes. Nous cherchons maintenant à étudier, dans un contexte plus large, le rôle et la contribution des différents acteurs (et actrices) du milieu au développement de la vie musicale durant la période de l'entre-deux-guerres. Les problématiques que cette période historique suscite sont nombreuses et ont souvent des liens entre elles. Quel rôle ont joué les femmes dans la diffusion de la musique canadienne et contemporaine? Quels ont été les enjeux nationalistes derrière la création de la Société canadienne d'opérette en 1922? Quelle a été la teneur du discours de Frédéric Pelletier, critique et chroniqueur musical au journal Le Devoir de 1917 à 1944? Que signifiait " composer » avant la création de la Ligue de compositeurs canadiens en 1951? Quelles ont été les conséquences de l'idéologie nationaliste sur les débats entourant la création du Montreal Orchestra (1930) et de la Société des concerts symphoniques (1934)?

La plupart de ces questions ont émergé durant la lecture des travaux de l'historien Yvan Lamonde, dont la dernière étude sur l'histoire sociale des idées au Québec présente une admirable synthèse des recherches qu'il a accomplies depuis plus de vingt ans «pour rendre compte du circuit complet des idées, de leur production, de leur diffusion, de leur réception ». Deux champs de recherche ont cependant été exclus de cet immense chantier : l'art en général et particulièrement la musique de concert, et les institutions culturelles de la communauté anglophone, l'auteur souhaitant laisser à d'autres « le goût de nouveaux clearings... ${ }^{1}$.

1 Yvan Lamonde, Histoire sociale des idées au Québec (1760-1896) (Montréal : Fides, 2000), 1:9-10. 
Du côté de la musicologie, on ne peut que déplorer l'absence presque complète d'études sur les activités musicales du milieu anglophone montréalais pour la période qui nous concerne. Et les travaux des historiens de la communauté juive et protestante (qui auraient pu nous offrir quelques pistes) ont peu souligné, même à grands traits, l'implication de ces groupes dans le milieu musical'2. Que savons-nous de l'activité professionnelle et des propos sur la musique de Clara Lichenstein, Georges Brewer, Saul Brant, Stanley Gardner, Lynwood Farnam, Sarah Fisher, Ethel Stark, Douglas Clarke, pour ne nommer que les musiciens anglophones les plus connus?

Et même nos propres questions ne concernaient que l'histoire en milieu francophone jusqu'à ce que nous découvrions par hasard, dans les archives du musicien québécois Rodolphe Mathieu, un programme d'une société dont nous ignorions l'existence ${ }^{3}$. Ce document d'une cinquantaine de pages relate les activités d'une Semaine annuelle de musique (SAM) qui eut lieu en février 1929 sous le patronage d'une association féminine anglophone, le Delphic Study Club (DSC), durant laquelle Mathieu sollicita quelques musiciens pour présenter des pièces musicales de ses étudiantes en composition. Un geste audacieux et historique puisqu'il s'agissait du tout premier concert montréalais d'œuvres de compositrices ${ }^{4}$. Nous avons découvert par la suite que cet événement annuel avait eu lieu durant quinze ans, soit de 1923 à 1937. En raison même de cette durée, nous considérions que le sujet méritait une analyse plus approfondie.

Toutefois, nos démarches pour en connaître davantage sur le DSC se sont avérées jusqu'à ce jour infructueuses. Il ne semble subsister aucunes archives et même l'identité de la plupart des directrices demeure encore un mystère. Fondé par $\mathrm{M}^{\mathrm{me}}$ Elizabeth Griswold Waycott, ce club a tenu l'un de ses premiers déjeuners-causeries le 16 octobre 1919 au Ritz-Carlton, présidé par $\mathbf{M}^{\text {me }}$ Joseph E. Duval. La conférencière invitée, $\mathbf{M}^{\text {me }}$ Adelia Potter, de New York, parle du sens de l'organisation que doivent développer les femmes pour faire valoir leurs droits. Puis $\mathbf{M}^{\mathrm{me}} \mathrm{J}$. E. Armstrong explique de son côté l'histoire de l'oracle de Delphes, à l'origine du nom du club.

The importance of organization was the keynote of a talk on woman suffrage given yesterday at the first luncheon of the Delphic Study Club at the Windsor Hotel by Miss Adelia Potter of New York. That the women of New York would not have won the vote had there not been excellent organization ${ }^{5}$.

2Entre autres, les nombreux travaux de Pierre Anctil sur la communauté juive et l'ouvrage de Margaret W. Westley, intitulé Grandeur et déclin : l'élite anglo-protestante de Montréal 1900-1950 (Montréal : Libre Expression, 1990).

3 Fonds de la famille Mathieu, Bibliothèque nationale du Canada, MUS 165/A. Les programmes des années 1931 et 1932 ont été publiés dans B. K. Sandwell, The Montreal Music Year Book (Montréal : Montreal Music Year Book Registered, (1931) : 49-56; (1932) : 9-19.

4 « Something decidedly out of the ordinary ", écrit un critique anonyme dans The Gazette, 22 mars 1929, 12. Rappelons que la compositrice Albertine Morin-Labrecque a organisé un Festival de ses propres œuvres le 24 mars 1935.

5 «L'importance d'une organisation efficace a été l'élément majeur de la conférence sur le suffrage féminin présentée hier au premier déjeuner du Delphic Study Club à l'hôtel Windsor par $\mathrm{M}^{\text {me }}$ Adelia Potter de New York, car, a-t-elle dit, les femmes de New York n'auraient jamais gagné ce vote n'eut 
Durant quinze ans, les journaux font référence à ce club comme si sa présence dans la communauté culturelle allait de soi; les critiques ne mentionneront tout au plus que le nom (probablement celui de leur époux), et parfois le prénom des membres. Seul le journal La Presse, qui consacre plus d'une dizaine de pages à la SAM de 1925, reproduira l'unique photographie représentant les sept organisatrices de cette saison ${ }^{6}$. Contrairement à d'autres associations mieux connues, comme le Ladies Morning Musical Club ${ }^{7}$ ou le Toronto Women's Literary $\mathrm{Club}^{8}$ qui organisaient des activités uniquement pour leurs membres, le Delphic Study Club, représenté par une dizaine de personnes, a présidé la supervision d'une Semaine annuelle de musique dans le milieu montréalais. Les membres établissaient les contacts avec l'industrie musicale, les postes radiophoniques, les hôtels, les différentes associations communautaires et les institutions scolaires, et déléguaient ensuite aux organismes ainsi sollicités un responsable (issu du milieu musical) qui veillait à la programmation des concerts; à ce titre, plusieurs musiciens francophones furent invités à participer.

Cette SAM a retenu notre attention car elle représentait une première initiative dans le domaine de l'éducation musicale du grand public et une première tentative de coopération entre les deux communautés linguistiques montréalaises. Nous avons donc procédé, avec la collaboration de quelques étudiants, à l'inventaire des données recueillies dans la presse ${ }^{9}$. L'analyse du discours tenu durant ces activités annuelles fait ressortir des divergences importantes quant à la conception et au rôle de la musique dans la société, et met en relief le regard nationaliste que la critique musicale francophone porte sur l'événement.

Présentons d'abord cette Semaine annuelle de musique qu'évoquait ainsi la pianiste Gilberte Martin :

Nous pouvions jouer jusqu'à quatorze fois en cinq jours : le matin à l'école Fairmount pour les jeunes, l'après-midi dans les petits salons du Windsor et

été la mise en place d'une excellente organisation. » "Women's Work », The Montreal Star, 17 octobre 1919, 12; 28 janvier 1920, 13. À moins d'avis contraire, les traductions sont de l'auteure du présent article.

Nous remercions Carl Morey, responsable de la chaire Chalmers en musique canadienne de l'Université de Toronto, pour ses recherches sur l'existence d'un DSC en Ontario et pour nous avoir confirmé que ce club ne semble avoir existé qu'au Québec.

6 « Semaine de la musique », La Presse, 28 mars 1925, 29-38.

7 Cette vénérable institution attend toujours qu' on écrive son histoire.

8Heather Murray, « Great Works and Good Works: The Toronto Women's Literary Club, 1877$1883 »$, Historical Studies in Education $11, \mathrm{n}^{\circ} 1$ (printemps 1999) : 76-98.

9Helmut Kallmann, Gilles Potvin et Kenneth Winters, édit., Encyclopédie de la musique au Canada (Montréal : Fides, 1993). Cet ouvrage de référence ne mentionne que deux fois la SAM, aux entrées " Festivals " (1:1177) et "Canadian Bureau for the Advancement of Music » (1:484). Je remercie Raquel Bontempo, Chantal Denis, Madeleine Lemieux, Sonia Pâquet, Raymond Lepage, Tam-Ca Vo et Frédéric Samson pour leur collaboration à ce projet, ainsi que Claudine Caron qui a produit la synthèse des données. Je remercie également le Conseil de recherches en sciences humaines du Canada et l'Université de Montréal pour leur appui financier. Nous avons procédé à la collecte des données dans les périodiques suivants : La Presse, Le Devoir, La Patrie, The Gazette, The Montreal Daily Star, The Montreal Herald, La revue Passe-Temps et La Lyre, pour la période de 1918 à 1938. 
du Mont-Royal pour les dames; et le soir, dans les somptueuses salles de bal de ces grands hôtels, pour le grand monde [...]. C'était extrêmement stimulant et d'une récompense inestimable de voir des salles entières de vrais amateurs de musique nous suivre et nous encourager d'un hôtel et d'un concert à l'autre ${ }^{10}$.

\section{ORIGINE, STRUCTURE, MODE DE FONCTIONNEMENT}

C'est à l'hôtel Windsor en novembre 1922, lors d'un déjeuner-causerie sous la présidence de $\mathrm{M}^{\mathrm{me}} \mathrm{J}$. E. Armstrong, que des membres du DSC, composé de femmes à l'esprit ouvert et progressistes qui voulaient contribuer de façon concrète au développement culturel ${ }^{11}$, décident d'organiser, au printemps suivant, une Semaine annuelle de la musique où seront offerts gratuitement à la population des concerts tant dans les salles huppées des différents hôtels et magasins de musique de Montréal que dans les écoles, églises et autres lieux publics. Sous la devise officielle bilingue "Give more thought to Music Donnez plus de pensées à la musique », le DSC veut à la fois soutenir les jeunes talents et offrir aux citoyens la possibilité « d'embellir leur existence avec de la bonne musique $\gg^{12}$. Au cours de la première rencontre, la pédagogue américaine E. Hope McLea présente le Virgil Clavier Method, une méthode d'apprentissage du piano très répandue aux États-Unis au début du siècle. Elle sera invitée durant trois années consécutives $(1923,1924$ et 1925) aux journées d'ouverture de la SAM.

Le mode de fonctionnement de cette Semaine s'inspire d'une idée américaine implantée depuis peu par Charles Milton Tremaine (1870-1963), président d'une compagnie de fabrication de pianos de New York. Il fonde en 1916 le National Bureau for the Advancement of Music et crée en février 1920 The New York Music Week où participent plus de 1700 organisations musicales ${ }^{13}$. S'appuyant sur une conception bien victorienne de la musique, c'est-à-dire une musique de bon ton " considered as a pleasure to be universally enjoyed » [« considérée comme un plaisir partagé universellement»], dont la principale fonction est de maintenir des rapports familiaux et sociaux harmonieux et de faciliter une meilleure production dans les milieux de travail, Tremaine propose de faire du New York Music Week un mouvement national. Il affirme qu'une nation est qualifiée de « musicale » seulement lorsque toute sa popula-

10Cité dans Nicole Deslauriers, Si mon père m'était conté (Ottawa : Édicompo, 1982), 99.

11 « Composed of broaded-minded and progressive women who take practical interest in cultural matters and who have turned their attention especially to music ». Philipp King, « Music Week Plans », The Montreal Daily Star, 4 novembre 1922, 36. Parmi les invitées, se trouvaient : $\mathrm{M}^{\text {mes }}$ Jeffrey Burland, Arthur Léger, Edmond Guillet, Eugene Cowles, W. G. MacNaughton et [?] Harrison.

12 « Le pourquoi d'une Semaine de musique », Programme de la septième Semaine annuelle de musique, archives de Rodolphe Mathieu, fonds de la famille Mathieu, Bibliothèque nationale du Canada, MUS 165/A5,1. Ces Semaines eurent lieu aux dates suivantes : 11-17 mars 1923; 16-22 mars 1924; 28 mars -4 avril $1925 ; 4-10$ avril $1926 ; 3-10$ avril $1927 ; 25-31$ mars $1928 ; 17-24$ mars $1929 ; 5-12$ avril 1930; $23-30$ mars $1931 ; 13-19$ mars $1932 ; 2-8$ avril $1933 ; 19-24$ mars $1934 ; 7-13$ avril $1935 ; 30$ mars -4 avril 1936; 14-20 mars 1937.

13L'événement est annoncé dans l'article «Music and Musicians », The Montreal Star, 17 janvier $1920,17$. 
tion réagit à la musique et non pas parce que le milieu produit quelques grands musiciens :

We need the great musicians to lead us to discover the hidden beauties of music, but what the musicians need is that the people be made receptive followers so that they may be led to seek what the great composers and interpreters of music have to give. Great concert audiences are as essential as great artists [...]. New York should become the world's music center [...]. It needs a musical atmosphere which is alone the result of the influence of music upon the sum total of the people ${ }^{14}$.

Poursuivant cet objectif de développer un public mélomane (music lovers), il reçoit l'appui inconditionnel du critique musical du New York Globe, Charles David Isaacson ${ }^{15}$, qui viendra présenter le projet américain à Montréal lors de l'ouverture officielle de la Semaine en 1923.

Tremaine crée la National Music Week en mai 1924, à laquelle participent plus de 838 villes américaines; à la manière des grandes commémorations religieuses de Noël et de Pâques, puis des fêtes patriotiques (Fourth of July, Memorial Day, Thanksgiving Day, Armistice Day), Tremaine souhaite ainsi créer un rassemblement national qui serait officialisé par une proclamation présidentielle ${ }^{16}$. Et, affirmant que " the greater the circle of those who derive relaxation, pleasure and entertainment from music, the greater will be the encouragement to the composer, the artist and indeed to all professionally connected with music $»^{17}$, il parvient à convaincre le président américain de l'époque, Calvin Coolidge, de présider le comité d'honneur.

Si les organisatrices du Delphic Study Club choisissent d'implanter le modèle américain de Tremaine à Montréal, c' est qu' elles partagent cette vision utilitaire de la musique et trouvent, dans ce modèle d'organisation, une parenté

14 « Nous avons besoin des grands musiciens pour nous amener à découvrir les beautés cachées de la musique, mais ces musiciens ont aussi besoin que le peuple soit formé à devenir des serviteurs réceptifs afin de connaître ce que les grands compositeurs et interprètes ont à offrir. De larges auditoires sont aussi essentiels que les grands artistes. New York doit devenir le centre mondial de la musique. Pour cela, il est nécessaire de créer une ambiance musicale qui, seule, résulte de l'influence de la musique sur l'ensemble de la population. » C. M. Tremaine, History of the National Music Week (New York : National Bureau for the Advancement of Music, 1925), 27-28. Dans sa thèse intitulée « The Ascendancy of Musical Modernism in New York City, 1915-1929» (thèse de doctorat, Yale University, 1993), David J. Metzer consacre les premiers chapitres au concept de "genteel music ", cette musique « de bon ton » propre à la mentalité victorienne et qui caractérise le goût d'une certaine bourgeoisie américaine de l'époque.

15 Auteur, entre autres, d'un volume intitulé Face to Face with Great Musicians (New York : Appleton, 1921).

16Il évoque même la possibilité d' une Semaine internationale de la musique. Tremaine, History of the National Music Week, 24.

17 «Plus le cercle de ceux qui cherchent détente, plaisir et divertissement dans la musique sera grand, plus l'encouragement aux compositeurs, interprètes et professionnels de la musique sera important. " Tremaine, History of the National Music Week, 14. Dans sa conférence prononcée lors de l'ouverture de la $13^{\mathrm{e}}$ Semaine, le critique musical montréalais H. P. Bell mentionne qu' une des raisons pour lesquelles la Semaine américaine ne fonctionne plus en 1935 est peut-être parce que chaque semaine y est maintenant devenue musicale. H. P. Bell, « Music Week Given Official Opening ", The Gazette, 9 avril 1935, 3. 
familière spécifique au milieu anglophone : l'interaction naturelle entre le milieu des affaires et le milieu culturel, d'une part, et, d'autre part, l'esprit coopératif qui anime le bénévolat des femmes, un bénévolat construit sur l'idée d'une action à mener pour le bien communautaire et pour le développement de la nation. Ce qui fera dire au critique du journal Le Devoir, Frédéric Pelletier : "C'est à la coopération [entre organisateurs anglophones et musiciens francophones] qu'a eu recours le DSC et c'est grâce à elle qu'il devrait réussir ${ }^{18}$. »

\section{Collaborateurs}

L'organigramme de la SAM révèle une structure fonctionnelle et efficace qui comprend une présidente, entourée d'une vice-présidente, d'une secrétaire et d'une trésorière ${ }^{19}$. Ce Conseil agit uniquement au niveau de l'organisation générale et intervient auprès des gens d'affaires pour solliciter leur collaboration et un soutien financier afin d'offrir des bourses. Une directrice générale coordonne la logistique avec les principaux établissements choisis et nomme un responsable artistique, musicien ou musicienne, pour chaque organisme qui accepte de participer au projet (écoles, églises, chorales, ensembles instrumentaux, organismes musicaux, classes d'élèves de professeurs privés, conservatoires et écoles privées, salles de concert des maisons de vente de pianos) ${ }^{20}$. Ces responsables de la programmation, d'abord anglophones féminins, deviennent majoritairement francophones masculins entre 1929 et 1934, surtout lors des concerts " professionnels » offerts dans les salles plus prestigieuses des hôtels de Montréal. L'ensemble des activités est couronné par les concerts et conférences d'ouverture et de clôture sous la direction du Conseil.

18Frédéric Pelletier, «La Semaine musicale ", Le Devoir, 10 mars 1923, 6.

19Plusieurs présidentes se sont ainsi succédées durant ces quinze ans : $\mathbf{M}^{\text {mes }}$ Elizabeth GriswoldWaycott (1922, 1924), J. E. Armstrong (1923), Rose McMillan (1925), Elizabeth Loudon (1926), Ellen G. Lawrence (1927, 1928, 1929), G. R. Williams (1930), D. C. Drysdale (1931), H. M. Christmas (1932, 1934, 1935), F. E. Melkman (1933), Maud Ellis-Starr (1936, 1937).

20Parmi les collaborateurs, mentionnons : Académie du Québec. Ampico (disques), Association for the Blind, Baron de Hirsch Book Club, Canadian National Railways Radio Station in Montreal (CNRM), Carabiniers du Mont-Royal, Catholic Women's League, Cercle paroissial de Saint-EnfantJésus, Club des femmes de NDG, Club des femmes juives, Club des jeunes filles suisses, Club Kiwanis, Collège canadien des organistes, Collège de musique de Montréal, Conservatoire national de musique, Conservatoire Racicot, Côte-des-Neiges Women's Club, École de musique de Montréal, Heintzman \& Co., École supérieure de musique d'Outremont, Emmanuel Young People's Society, Homemaker's Club of the Settlement, International Music Store, Killarney Girl's Club, Ladies' Morning Musical Club, Lakeside Citizen's Association, Ligue des femmes catholiques, Lion's Club, Local Council of Jewish Women, Marconi Station (CFCF), Matinee Musical Club, McGill Musical Club, Mercury Club, Metropolitan Choral Society, Montreal West Ladies' Glee Club, Montreal West Women's Club, Montreal Women's Club, Mount Royal Women's Community Club, Nazareth Institute for the Blind, North End Women's Club, Old Country Club, Rotary Club, Saint-Lambert Women's Club, Salvation Army, Société canadienne d'opérette, Société philharmonique de Montréal Est. Station radiophonique CHYC, Station radiophonique CKAC, Station radiophonique CKNE (Toronto), Temple Emmanuel Sisterhood, Thursday Morning Musical Club, La Voix de son Maître (disques), Westmount Women's Club, Women's Guild, YMCA Central, Young Men's Hebrew Association. 


\section{COMMANDITAIRES ET BOURSES}

Jusqu'à la création du Conservatoire de musique du Québec en 1942 (conçu selon le modèle européen, c'est-à-dire gratuit, laïc et géré par l'État), et malgré l'absence au Québec d'une culture de mécénat, le gouvernement a très peu soutenu le milieu musical. En fait, on ne retrouve que le Prix d'Europe pour les interprètes à partir de 1911, quelques bourses pour les compositeurs émanant du bureau du Secrétaire de la Province (1920-1928), une aide ponctuelle au Conservatoire national de musique en 1930 (une entreprise privée qui s'associera par la suite au réseau américain des Gregorian Institutes où l'on enseignait la musique liturgique), un soutien à l'émission radiophonique de CKAC, L'Heure Provinciale (1929-1939), et un appui limité à la Société des concerts symphoniques fondée en 1934. Cette aide favorisait avant tout la formation des interprètes, mais pour ce qui est de l'éducation musicale du public, tout semble s'être réalisé " à la manière américaine », par une collaboration étroite entre les marchands de musique et le bénévolat féminin. Ainsi, durant cette Semaine, billets, salles, instruments et musiciens étaient offerts gratuitement grâce aux commandites de l'industrie musicale et plusieurs accorderont de modestes bourses en chant, piano, violon, violoncelle et orgue, à partir de $1925^{21}$. Rappelons quelques boursiers du DSC qui firent carrière par la suite : Jean Deslauriers et Ethel Starke (1926); Paul-Émile Corbeil, Gilberte Martin et Noël Brunet (1928); Bernard Piché, Gérald Desmarais, Cécile Préfontaine et Jean Papineau-Couture (1930); Mildred Goodman (1932); et Rosette Renshaw (1937).

\section{LIEUX, AUDITOIRES, CONCERTS, INTERPRÈTES}

L'un des débats les plus vifs auquel a donné lieu la SAM portait sur les nombreux endroits où avaient lieu les concerts, presque exclusivement dans l'ouest de la ville où vivait une majorité anglophone, ainsi que dans certaines banlieues périphériques dont Lachine et Saint-Lambert ${ }^{22}$. Cette question de la

21 Parmi les commanditaires de l'industrie musicale, nous relevons les noms suivants (trois magasins de piano qui offraient une salle de concert sont mis en évidence) : Bagdad (salle de danse devant l'hôtel Mont-Royal), C. A. Beaudet (radio), Dantes Belleau (professeur), $\mathrm{M}^{\text {lle }}$ E. Bonhomme (professeur), Canadian Gramophone \& Piano Limited, Canadian National Railways, Charles Lavallée Enr., Chez Jos. Lagarde, Cie Royale de pianos et phonographes, Édouard Cusson (accordeur), C. W. Lindsay Ltd., Davies Studios Ltd., Dominion College of Music, Dupuis Frères, Échange Mont-Royal, École de musique Drouin, École de violon Camille-Couture, Edmond Archambault Enr., Fairweather, Hartney Co. Ltd., Heintzman \& Co., Henry Gatehouse \& Son, His Master Voice, Hôtel Queen's, J. A. Ogilvy's Ltd., J. Donat Langelier Ltée, Kerhulu \& Odiau Ltée (restaurant du Quartier Latin), Layton Bros. Ltd., Leo Feist Ltd., J. J. Levert, Maison Pinet et Jarry, Marconi Radios, Michaud \& Cie Ltée, Northern Electric Co. Ltd., Paquin \& Cie, Peate Musical \& Co., Le Phonographe Pathé Enr., J. O. Pilon, Shaw Memorial Junior Choir, Shaw's Radios, Star Phonograph Co., J. E. Turcot, Raoul Vennat, V. Laroi Ltd., White Star Dominion Line, Willis \& Co.

22Écoles : Aberdeen, Académie Querbes, Académie St-Patrice, Academy Stratcona, Alexandra, Alfred Joyce, Argyle, Bancroft, Barclay, Belmont, Central High School, Collège Notre-Dame, Commercial High School, Connaugh, Devonshire, Dufferin, Earl Grey, École Berthelet, École de Cartierville, École du Doux Parler, École protestante des aveugles, Edward VII, Fairmount, Gilson, Hamilton, Herbert Symonds, Kensington, King, Lansdown, Lorne School, Maisonneuve, McVicar, Montreal High School, Mont-Royal, Peace Centennial School, Prince Albert, Queen's, Riverside, Rockland, Rosemont, Roslyn, Royal Albert, Royal Arthur, Saint-Patrick Academy, Sarah Maxwell Memorial, Strathearn, 
localisation de lieux de concerts pouvant desservir les francophones était déjà à l'ordre du jour lors de la création du Parc Sohmer en 1889 et du Monument National inauguré en 1893. Le critique musical du journal nationaliste Le Devoir, Frédéric Pelletier, reprend le débat sur l'urgence de construire une salle de concert dans l'est ${ }^{23}$, tout en questionnant le milieu scolaire francophone qui refuse, pour des questions linguistiques et religieuses, d'ouvrir les portes de ses écoles aux organisateurs des concerts.

Mais qu'en est-il de la participation du public? Si on se fie aux plaintes fréquemment rapportées dans les journaux où il est fait mention de personnes refusées en grand nombre, faute de places, on peut en déduire que le taux de fréquentation fut élevé, tant dans les lieux plus prestigieux (théâtres, hôtels ou maisons de vente de pianos) que dans les écoles. Par contre, durant ces quinze ans d'activités, nous n'avons pu relever de données précises sur les caractéristiques de l'auditoire, aucun critique n'ayant laissé de témoignage à ce sujet. Seul Léo-Pol Morin estime, en 1927, à environ 50000 le nombre de personnes qui assistent annuellement à la $\mathrm{SAM}^{24}$. On peut cependant présumer que le public francophone participa largement si on se fie au nombre d'articles publiés par les journaux de cette communautées.

À défaut de pouvoir présenter une description des publics, nous pouvons, par contre, offrir une vision d'ensemble des activités musicales et des musiciens qui ont participé bénévolement à ces concerts entre 1923 et 1937. Le tableau suivant présente le nombre annuel de concerts, le profil des interprètes

Victoria, WestHill High School, Westmount High School, William Dawson, William Lunn.

Églises : American Presbyterian Chapel, Centenary Church (Pointe Saint-Charles), Christ Church Cathedral, Church of the Messiah, Église de l'Immaculée-Conception, Église Melville, Église méthodiste de NDG, Église Sainte-Anne, Emmanuel Temple, Erskine Presbyterian Church, Kensington Presbyterian Church, Methodist Temple, Rosemont Central United Church, Shaar-Hashomayin Synagog, Shaw Memorial Methodist Church, St. Andrew Church, St. Henry Methodist Church, St. James Methodist Church, St. Stephen Church (Lachine), Wesley Methodist Church, West End Methodist Church, West Montreal Presbyterian Church.

Lieux communautaires : Arsenal des Carabiniers Mont-Royal, Brawery Mission, Citadel Hall, Congress Hall, Country Club House, Griffin Town Club, Hôpital militaire Sainte-Anne, Hôtel de ville de Lachine, Hôtel de ville de Maisonneuve, Hôtel de ville de Pointe-aux-Trembles, Hôtel de ville de Pointe-Saint-Charles, Hôtel de ville de Saint-Lambert, Institut du Baron de Hirsch (rue Bleury), Iverly Settlement, Kings of Colombus Hall, McGill Union, Montreal West Town Hall, Rotary Club, Salle Saint-Sulpice, Salvation Army Citadel, Sanatorium de Sainte-Agathe, Town Mount-Royal Clubhouse, Trinity Memorial Hall, University Settlement, Victoria Hall, Westmount Community Center, YMCA (centre-ville), YMCA (nord).

Théâtres : Allen, Auditorium le Plateau, Empress, Imperial, Lachine, Loew's, Palace, Princess (appartenant à la maison Willis), Régent, Royal Alexander, Saint-Denis.

Hôtels : Mont-Royal, Queen, Ritz Carlton, Wilhelmina, Windsor (salle Ladies' Ordinary).

Maisons de vente : salle Langelier, salle Layton, salle Lindsay, salle Michaud, salle Tudor, salle Willis.

Ajoutons à cette liste le poste radiophonique CKAC qui diffusait les concerts des boursiers et la maison d'enregistrement His Master Voice Hall qui proposait au public des auditions de disques.

23Frédéric Pelletier y fera constamment allusion durant toute sa carrière au journal Le Devoir, de 1917 à 1944. Ce n'est qu'en 1963 que la salle Wilfrid-Pelletier de la Place des Arts ouvrira finalement ses portes.

24Léo-Pol Morin, « La Semaine de musique », La Patrie, 9 avril 1927, 34.

25Le journal La Presse y consacre plus de 9 pages en 1925. 
ainsi que le nombre de groupes musicaux (incluant musique de chambre, chorales, harmonies) qui se sont produits.

Tableau 1 : Concerts et interprètes participant annuellement à la SAM

\begin{tabular}{|c|c|c|c|c|c|c|c|c|}
\hline \multirow[b]{2}{*}{ Année } & \multirow[b]{2}{*}{ Concerts } & \multicolumn{3}{|c|}{ Interprètes féminines } & \multicolumn{3}{|c|}{ Interprètes masculins } & \multirow[b]{2}{*}{ Groupes } \\
\hline & & Anglophones & Total & Francophones & Anglophones & Total & Francophones & \\
\hline 1923 & 23 & 93 & 123 & 30 & 66 & 87 & 21 & 10 \\
\hline 1924 & 36 & 54 & 93 & 39 & 30 & 71 & 41 & 23 \\
\hline 1925 & 43 & 53 & 124 & 71 & 30 & 80 & 50 & 17 \\
\hline 1926 & 49 & 109 & 165 & 56 & 59 & 111 & 52 & 17 \\
\hline 1927 & 60 & 46 & 90 & 44 & 31 & 75 & 44 & 13 \\
\hline 1928 & 57 & 52 & 93 & 41 & 44 & 83 & 39 & 9 \\
\hline 1929 & 50 & 51 & 109 & 58 & 28 & 74 & 46 & 16 \\
\hline 1930 & 48 & 29 & 74 & 45 & 27 & 65 & 38 & 6 \\
\hline 1931 & 36 & 27 & 73 & 46 & 15 & 65 & 50 & 10 \\
\hline 1932 & 26 & 31 & 82 & 51 & 21 & 68 & 47 & 7 \\
\hline 1933 & 24 & 12 & 46 & 34 & 7 & 30 & 23 & 13 \\
\hline 1934 & 17 & 30 & 94 & 64 & 18 & 55 & 26 & 3 \\
\hline 1935 & 9 & 19 & 73 & 54 & 9 & 39 & 31 & 5 \\
\hline 1936 & 9 & 25 & 46 & 21 & 32 & 36 & 4 & 5 \\
\hline 1937 & 9 & 29 & 57 & 28 & 22 & 35 & 13 & 2 \\
\hline Total & 496 & 660 & 1342 & 682 & 439 & 974 & 525 & 156 \\
\hline
\end{tabular}

La plupart des journaux rapportent fidèlement la programmation proposée par le comité organisateur en indiquant le lieu, la date et l'heure, ainsi que le nom du responsable artistique. Les années fastes de cette Semaine se situent entre 1924 et 1931. L'augmentation rapide du nombre de concerts, passant de 43 à 60 entre 1925 et 1927, s'explique par le nombre de plus en plus important d'ententes avec le réseau scolaire protestant qui bénéficiera de la présentation de plusieurs événements. Par ailleurs, sauf exception, seuls les concerts mettant en vedette des interprètes professionnels ou semi-professionnels, et présentés dans des salles renommées ou diffusés à la radio, ont publié leur programme dans les journaux. Les chiffres concernant les interprètes et les groupes musicaux inscrits au Tableau 1 ne représentent que ces concerts plus officiels. Les statistiques demeurent muettes sur le contenu et le nombre d'interprètes présentés dans les écoles ou par les classes d'élèves de professeurs reconnus ${ }^{26}$. Il est à noter que chaque récital de clôture offert par les boursiers a été radiodiffusé par le poste CKAC.

En ce qui concerne la représentation des instruments, seuls le chant, le piano et le violon (et exceptionnellement le violoncelle et l'orgue, surtout chez les interprètes masculins) ont été privilégiés lors de ces concerts, probablement parce qu'une grande partie de ceux-ci, donnés par des élèves, cherchaient à mettre en valeur l'enseignement privé des professeurs. Par contre, la répartition

26Sauf pour l'année 1926 au cours de laquelle les journaux anglophones ont publié, exceptionnellement, le détail des concerts donnés dans les écoles et les lieux communautaires. C'est ce qui explique le nombre plus élevé d'interprètes féminines anglophones cette année-là. 
des données entre les musiciens montrent un taux de participation légèrement plus élevé chez les francophones, particulièrement parmi les interprètes masculins, ce qui peut étonner compte tenu du caractère linguistique de l'entreprise et de l'indifférence des autorités locales envers la SAM.

Plusieurs ensembles ont eu l'occasion de se produire lors de ces concerts : chorales, orchestres, harmonies et quatuors à cordes. Alors que l'histoire n'a retenu que quelques-uns d'entre eux (le Quatuor Dubois et le Trio Lamontagne, par exemple), on constate qu'un nombre important d'ensembles ont animé la vie musicale de l'époque. Outre les nombreux quatuors vocaux, on relève les groupes instrumentaux suivants : le Trio César Frank (Leon Koftman, Ernest Gagnier, Gilbert Hill), le Trio de l'Hôtel Windsor (Harry Salter, Raoul Duquette, Harry Lipman), le Trio du Bon Vieux Temps (M ${ }^{\text {lles }}$ Forest, Pagé et Cabana), le Trio Desrochers (J. J., R. A. et Félix Desrochers), The Feminine String Quartet ( $\mathbf{M}^{\text {lles }}$ F. Parker, E. Marshall, F. Smythe et F. Melin), le Trio Muriel Pitts (Mlles $Y$. Dalpé, M. Wynn, M. Pitts), le Trio et le Quatuor Jean Deslauriers, le Quatuor féminin Arcand (Kathleen, Marie, Madeleine et Marguerite Arcand), le Trio Choquette (Thérèse et Gabrielle Choquette, Yvonne Lefebvre), le Quatuor à cordes Gaudriot (Louis Gaudriot, Stanley Widman, Jules Lapointe et $\mathbf{M}^{\mathrm{me}}$ Foster McNames), le Trio Leo Ashby, le Wynn Ladies' Trio, le Trio Leduc (Anne Lasalle, Roland et Jean Leduc), le Quatuor Nocturne (Jean Deslauriers, Henri Brunet, Jean Robert et Paul Bourgeois), le Montreal Ladies' String Quartet, le Oxford Ladies' Quartet (Margaret Douglas, Vera Livershidge, Gras Cowan et Audrey Hutchison). Soutenir, comme nous l'avons déjà entendu, que la vie musicale au Québec n'était qu'un pur désert avant 1950, apparaît donc fort discutable...

\section{LE RÉPERTOIRE}

Philip King rapporte, en 1923, que les programmes proposés ne s'adressent pas aux musiciens mais plutôt à un large public :

These programs are not made primarily for musicians like the programs given at chamber music concerts, but are so constructed that the more general type of person known as « music lover» will not be frightened away by them ${ }^{27}$.

Dix ans plus tard, Thomas Archer fait le constat suivant :

Regarding the standard of music performed, a rough analysis of the programme of the week reveals the fact that about $50 \%$ of the pieces given at various concerts were first-class music, music by acknowledged masters. Pieces of a lighter nature but still good enough to grace any programme amounted to another $40 \%$, leaving only about $10 \%$ to be classed as indifferent from a cultural point of view ${ }^{28}$.

27 « Ces programmes ne s'adressent pas aux musiciens comme ceux qu'on retrouve aux concerts de musique de chambre mais sont préparés de façon telle qu'une clientèle plus générale, parfois nommée "amateur de musique", ne sera pas heurtée par le contenu musical. » Philip King, «Improving Taste », The Montreal Star, 10 mars 1923, 11.

28 « Concernant la qualité des programmes, $50 \%$ des œuvres jouées appartenaient au grand 
Il est vrai qu'une partie du répertoire renvoie à d'obscurs compositeurs ou encore à des pièces mineures de grands maîtres et on ne peut que déplorer le goût conservateur des professeurs qui préfèrent enseigner de courtes pièces de virtuosité29. Même si certains musiciens plus aventureux proposèrent des œuvres du répertoire moderne, dont celles de Debussy ${ }^{30}$, le choix fut souvent dicté par l'industrie de l'édition musicale qui adhérait au discours moralisateur tenu par les autorités sur la fonction de la musique (comme moyen de préserver les valeurs familiales), et ignorait toute création ne correspondant pas à cette genteel music ${ }^{31}$. Plusieurs concerts de grande qualité ont ainsi été noyés dans le flot des activités de la Semaine, jusqu'à ce que les plaintes répétées des critiques musicaux incitent les membres de la SAM à rectifier le parcours, à partir de 1931, en offrant davantage de concerts professionnels et moins de récitals scolaires. Quant au répertoire canadien, la SAM n'est pas au rendezvous. Seulement trois des 496 concerts ont été consacrés aux œuvres canadiennes; le premier eut lieu le 21 mars 1924; le second, le 21 mars 1929, au cours duquel Rodolphe Mathieu fit entendre des pièces de ses étudiantes en composition; et le troisième, le 18 mars 1932, organisé par le Conservatoire national de musique qui présenta quelques œuvres de ses professeurs ${ }^{32}$.

\section{CONFÉRENCIERS}

Deux conférenciers invités, représentant les deux communautés linguistiques, ouvrent et clôturent respectivement l'événement annuel. Et c'est peut-être dans leur propos que l'on perçoit avec le plus d'acuité les deux "solitudes » culturelles qu'évoque H. P. Bell dans le Montreal Star : " Above all things, co-operation is wanted. In music, as in many other things in Montreal, there are always too many people pulling in different directions without regard to

répertoire, un autre $40 \%$ était consacré à des œuvres plus légères mais bien écrites et un $10 \%$ représentait des pièces qu'on aurait avantage à oublier. " Thomas Archer, " Musical Topics ", The Gazette, 8 avril 1933, 10.

29Raymond Lepage constate que 60 pour cent des 159 noms de compositeurs recensés renvoient à d'obscurs créateurs qui n'ont pas traversé l'épreuve du temps. La Semaine annuelle de musique tenue sous les auspices du Delphic Study Club à Montréal du 4 au 10 avril 1926. Rapport de recherche (archives personnelles de l'auteure, juin 1999), 17-18. Il est permis de croire que ces professeurs étaient probablement sollicités par les magasins de musique qui leur fournissaient des échantillons de partitions en vogue.

30 C'est durant cette Semaine que le public put entendre le jeune André Laurendeau interpréter des œuvres de Debussy et de Chopin. Il participa comme pianiste aux concerts organisés par ses parents, Arthur Laurendeau et Blanche Hardy, les 17 mars 1929, 8 avril 1930 et 26 mars 1931. Charles Magnan commente ce dernier concert : " J'ai rarement entendu les "Minstrels" [Préludes, livre 1] de Debussy aussi originalement présentés. Ce jeune pianiste possède un talent extraordinaire mais se fie peut-être trop à sa facilité. » La Patrie, 27 mars 1931, 7.

31 « Only in the case of inferior music should a moral attitude be adopted [...]. Much modern music is neurotic music ", déclare le Révérend Lawrence Clare devant les membres du DSC. "Music and Morals Exist Separately », The Gazette, 26 mars 1934, 10.

32Notons que Charles Magnan, pianiste, compositeur et critique musical, à propos duquel nous ne connaissons que peu de choses, a présenté le 10 avril 1935 un poème symphonique intitulé L'Atlantique - composé de trois sections, « Chant marin », «Mer orageuse » et "Reflets bleus » - qu'il a lui-même interprété au piano. Il serait intéressant de retracer la partition de cette œuvre écrite par ce défenseur de la musique moderne. 
what others are doing ${ }^{33}$. » Un fossé sépare la vision esthétique protestante de la vision catholique sur l'importance de l'éducation musicale dans le réseau scolaire, sans parler des intérêts nationalistes et mercantiles qui s'y rattachent.

La première position, défendue successivement par les conférenciers Percival J. Illsley 1923, 1924 (organiste montréalais), J. A. Atkinson, 1923 (directeur du Bureau for the Advancement of Music, Toronto), G. A. Stanton, 1925 (surintendant musical pour les écoles protestantes), Sir Hugh Percy Allen (directeur du Royal College of Music de Londres), W. P. Percival (directeur général des écoles protestantes du Québec), Philip King et H. P. Bell (critiques musicaux au Montreal Star), T. Archer (critique musical au journal The Gazette) et A. P. Willis (propriétaire d'un magasin de musique), rappelle le rôle de l'éducation dans la formation du goût musical. Certains soulignent le rôle de la musique dans la vie familiale ( « the piano is a great consolidator of family life ») et dans la vie communautaire ( « to be better citizens if sometimes their daily lives were gladdened by some really good music $»)^{34}$. D'autres suggèrent $d$ 'ajouter aux cours de solfège déjà obligatoires dans les écoles anglophones des cours plus généraux sur les compositeurs et le répertoire ${ }^{35}$. Quant au clergé protestant, le Reverend Lawrence Clare rappelle que la musique « [...] is an expression of deep emotion by means of form, the feeling of communication it gives us ${ }^{36}$, et l'Archdeacon Gower-Rees souligne que la valeur de la musique « [...] is one of man's means of making contact with the greatest composer of all, God Himself ${ }^{37}$.

La seconde position est défendue presque uniquement par Frédéric Pelletier (dans ses allocutions de 1925, 1926, 1928, 1929, 1930 et 1931) et par Eugène Lapierre en 1933. Clérico-nationaliste convaincu, Pelletier souhaite dans un premier temps un meilleur équilibre des activités entre l'est et l'ouest de Montréal et conclut que ce partage d'une passion commune, la musique, conduira les deux races (selon l'expression de l'époque) à une meilleure compréhension ( music as a race link ») ${ }^{38}$. Mais dès 1925 il abandonne le débat sur l'éducation et se sert de cette tribune pour alerter l'opinion publique en faveur de la création d'une maison d'opéra, d'un orchestre permanent et surtout de la construction d'une salle de concert «dans l'est », projet sur lequel il revient annuellement ${ }^{39}$. Il propose même au DSC en 1928 de changer leur

33 «Ce qui est nécessaire avant tout, c'est la coopération. En musique, comme dans bien d'autres domaines à Montréal, trop de gens travaillent dans des directions opposées sans se préoccuper de ce que font les uns et les autres. » H. P. Bell, The Montreal Star, 17 avril 1926, 6.

34 «Le piano contribue de façon importante à resserrer les liens familiaux ». "Induce Public to Cultivate Music », The Gazette, 15 mars 1932, 6; « Les gens feraient preuve de plus'de civisme si la véritable bonne musique était intégrée à leur quotidien ». " Music Week », The Montreal Star, 5 avril 1930, 34.

35 Ces cours de solfège ont été mis en place dans les écoles protestantes par Guillaume Couture dès la fin du XIX ${ }^{\mathrm{e}}$ siècle.

36 «La musique exprime des émotions profondes et éveille un besoin de communication. » « Music and Morals Exist Separately ", The Gazette, 26 mars 1934, 10.

37 « La musique est l'un des moyens qui permet à l'homme d'entrer en contact avec le plus grand des compositeurs, Dieu lui-même. » « Music Is Declared Universal Speech », The Gazette, 6 avril 1936, 11.

38 « Tactful Methods with Non-Musical. Music Week's Success : Tribute to Efforts of Delphic Study Club : New Link for Two Races Suggested », The Gazette, 17 mars 1923, 7.

39Frédéric Pelletier, "Vie musicale », Le Devoir, 4 avril 1925, 6. 
devise pour " A Hall must be Built », bien éloignée du " Give more thought to Music ${ }^{40}$. Il suggère en 1929 de prendre modèle sur le système américain qui offre un bel exemple de la puissance engendrée par l'union des volontés et des capitaux expliquant que "l'Anglo-saxon [sic] est naturellement porté à la corporation tandis que le Latin est par tempérament plus individualiste ${ }^{41}$. Il annonce en 1931 la décision du gouvernement de construire une salle de concert de 3600 sièges grâce à un appui financier provincial et municipal de l'ordre de 25000 \$ par année durant 15 ans, financement obtenu par Eugène Lapierre suite à sa proposition d'acheter la Bibliothèque Saint-Sulpice pour y établir son Conservatoire ${ }^{42}$. Malheureusement, le projet n'ira pas plus loin, et l'année suivante, Pelletier ne peut que constater l'échec de la démarche.

\section{LA CRITIQUE MUSICALE}

De façon presque constante, les journaux anglophones endossent unanimement les objectifs poursuivis par le DSC dans de courts éditoriaux et se contentent de publier les programmes et d'afficher, en grand nombre, les annonces publicitaires. Chaque concert fait l'objet d'un bref compte rendu. La presse francophone, plus volubile et critique, publie photographies et articles sur la musique et les musiciens, et consacre de pleines pages, le samedi, aux activités de cette Semaine. Ces pages sur la musique deviennent même prétexte à émettre des opinions politiques.

Frédéric Pelletier s'indigne, le 10 mars 1923, que tous ces mouvements prennent leur origine exclusivement chez nos compatriotes de langue anglaise :

C'est un vigoureux mea culpa que nous devons battre sur nos poitrines. Puisque nous semblons incapables d'imaginer quelque chose, il ne nous reste qu'à nous rallier avec la volonté d'en profiter le plus possible. Soyons passifs puisque ce rôle nous convient et évitons le péché d'obstruction ${ }^{43}$.

Par ailleurs, il espère en 1924 que l'on fasse appel à plus de professionnels et à moins d'amateurs, et que les compositeurs canadiens soient mieux représentés. Il déplore que les autorités municipales soient absentes, alors qu'aux États-Unis le président donne publiquement son adhésion au mouvement. Et alors que le ministère de l'Instruction publique refuse de transmettre les noms des élèves au concours de Monsieur Atkinson, Pelletier fait volte-face en publiant les règles du concours et en invitant les étudiants à y participer ${ }^{44}$. Il souhaite en 1925 que les commissions scolaires francophones ouvrent leurs portes afin de décentraliser les activités de la $\mathrm{SAM}^{45}$. Il mentionne en 1926 que

\footnotetext{
40 « Music Week Is Officially Opened », The Herald, 27 mars 1928, 5; et « Montréal a besoin d'une belle salle pour ses concerts ", La Presse, 27 mars 1928, 17.

41 « Ouverture officielle de la $7^{\mathrm{e}}$ Semaine de musique », La Presse, 19 mars 1929, 8.

42 «L'ouverture de la Semaine annuelle de musique », La Quinzaine musicale, 28 mars 1931, 103 (texte intégral en anglais de l'allocution de Frédéric Pelletier).

43Frédéric Pelletier, «La vie musicale », Le Devoir, 10 mars 1923, 6.

44Frédéric Pelletier, "La vie musicale », Le Devoir, 8 mars 1924, 6; 15 mars 1924, 6; 22 mars $1924,3$.

45Frédéric Pelletier, «La vie musicale », Le Devoir, 28 mars 1925, 6.
} 
le succès de la SAM relève en grande partie de la présence de chanteurs et d'instrumentistes solistes aux dépens des chorales, et suggère en 1930 d'étaler les activités sur une année plutôt que de les concentrer à l'intérieur d'une seule semaine en invitant des conférenciers à présenter les œuvres; mais il se plaint en 1932 que cette semaine soit devenue une "débauche d'amateurisme " ${ }^{46}$.

Deux discours de sourds : les uns soulignant l'importance de développer des publics amateurs et l'influence de la musique sur le comportement social des individus, et les autres affirmant que le développement du goût va de pair avec le prestige d'une musique jouée par des professionnels. Quelques voix dissidentes s'élèvent au-dessus de la mêlée et remettent en question le bien-fondé de ces activités, de même que leur impact présumé sur l'avancement de l'art musical. On se demande même, à mots couverts, si l'objectif d'amener la musique aux moins nantis de la société est atteint, ou s'il ne répond pas plutôt à un impératif commercial dicté par l'industrie musicale.

George Brewer, musicien éclairé, réclame dès 1924 que le comité organisateur réunisse plus de 50 pour cent des musiciens professionnels ${ }^{47}$. L'année suivante, Arthur Letondal réagit à la conception même de la musique véhiculée par l'industrie : «Il faut faire comprendre au peuple que la musique est surtout un art et non un divertissement ou un agrément ${ }^{48}$. » En 1928 et 1929, Léo-Pol Morin s'en prend d'abord au bénévolat que demande le DSC aux musiciens, alors qu'il ne viendrait à l'idée de personne de solliciter les professions libérales ou médicales, et trouve pour le moins bizarre que ces musiciens soient confrontés à une obligation presque morale de se donner ainsi durant la SAM, « à administrer à forte dose de la musique à des gens qui ordinairement n'en réclament pas ${ }^{49}$. Puis, il questionne le choix du répertoire offert durant ces concerts :

Je n'ai jamais été partisan d'un art pour le peuple, ou plutôt d'un art qui soit susceptible d'intéresser tout le monde, sans distinction de culture, d'éducation, de condition ou de mœurs. Il n'est d'ailleurs pas souhaitable qu'un tel art existe et si certains chefs-d'œuvre sont appréciés de tous, il n'en demeure pas moins vrai que les uns et les autres les comprennent différemment. Je sais bien qu'il ne s'agit pas de révéler à notre peuple les beautés particulières et si dangereuses du Pierrot lunaire de Schoenberg ou des derniers Quatuors de Hindemith non plus que la beauté à la fois humaine et froidement plastique de certaines fugues de Bach. Je le croirais d'ailleurs, ce peuple, plus apte à comprendre ces chefs-d'œuvre ordinairement hermétiques pour la masse, que certaines musiques trop faciles dont il est abondamment servi pendant la semaine d'orgie sonore. [...] Mais si c'est du plaisir qu'on veut donner, qu'on en donne à tous les concerts. Mais alors ce n'est pas 60 concerts en 8 jours, c'est 60 beaux concerts au cours de l'année qu'il faut donner et on devrait

46Frédéric Pelletier «La vie musicale », Le Devoir, 17 avril 1926, 6; 5 avril 1930, 6; 26 mars 1932, 6.

47Lettre de George Brewer adressée au Montreal Star, 29 mars 1924, 5.

48 Arthur Letondal, «Placer la musique au rang des matières qui s'enseignent à l'école ». La Presse, 28 mars 1925, 29-30.

49Léo-Pol Morin, «La Semaine de musique », La Patrie, 24 mars 1928, 36. 
éviter surtout les concerts d'amateurs ou d'élèves non encore sortis de leur coquille ${ }^{50}$.

C'est à cette tâche que se consacrera Douglas Clarke. S'adressant aux membres du DSC en 1930, le nouveau doyen de McGill observe

[...] qu'il faut faire l'éducation musicale des enfants et non pas uniquement leur faire jouer d'un instrument ou cultiver leur voix. Ils doivent être instruits sur la musique, savoir l'écouter et connaître les auteurs. Pour cela il faut que l'enseignement de la musique soit au programme de toutes les écoles ${ }^{51}$.

Il ajoute : «Avez-vous travaillé uniquement pour flatter l'oreille d'indifférents qui viennent flâner une heure ou deux dans une salle de concert? Il serait beaucoup mieux d'avoir moins d'auditions et de rehausser leur niveau artistique. »

Réunissant des musiciens des deux communautés montréalaises, Clarke fonde en octobre 1930 le Montreal Orchestra. Sa mission : former un public mélomane en lui proposant, sur une base régulière, tous les dimanches de la saison artistique, l'audition d'œuvres du grand répertoire. Devant le peu d'appui financier que porta le milieu de l'industrie musicale à ce nouvel orchestre, Clarke fit appel à un comité de dames qui se chargea de solliciter des fonds. Lors du $100^{\mathrm{e}}$ concert, le 4 février 1935, il créa des Matinées pour les jeunes auditeurs.

\section{BILAN}

Les fondements mêmes de la SAM reposent sur une ambiguïté quant au rôle de la musique dans l'éducation, une idée défendue de façon bien différente par les milieux protestant d'un côté et catholique de l'autre. Lointaine héritière des réflexions de la Réforme et de la Contre-Réforme, la musique s'inscrit chez les uns comme un investissement rentable, qui se vit en famille, favorise la socialisation, sensibilise l'âme à la droiture et contribue à l'éducation du bon citoyen; alors qu'elle est considérée par les autres comme une activité élitiste, qui suppose des connaissances techniques instrumentales ou vocales pouvant mener à une activité professionnelle ${ }^{52}$, ce qui explique peut-être le peu d'intérêt

50Léo-Pol Morin, « La Semaine de musique », La Patrie, 16 mars 1929, 34.

51 « Douglas Clarke Spoke at Music Week Luncheon of the Delphic Study Club », The Montreal Star, 14 avril 1930, 6. Résumé du discours : Henri Letondal, «La Semaine de musique », La Patrie, 15 avril 1930,8 .

52Le thème d'un concours organisé en 1924 par le Canadian Bureau for the Advancement of Music durant la SAM témoigne bien de cette conception et combien ce thème pouvait être étranger aux préoccupations des éducateurs francophones à qui le Département de l'Instruction publique avait refusé la permission de communiquer les noms des élèves pour participer à ce concours : " The advantages in investments to make possible the culture enjoyment of music in the home, compared with investments that offer chiefly pleasure. " « Prize Competitions for Essays on Music », The Montreal Star, 15 mars 1924, 9.

Dans un autre ordre d'idées, lors d'une conférence devant des gens d'affaires au Mount Royal Lodge, le 10 avril 1930, Frédéric Pelletier, plaidant en faveur de la création d'un orchestre permanent. rappelle une citation du richissime président du Metropolitan Opera, Otto Khan : "Those who have made their money with the money of others owe them dividends, not in money but in elevating their soul with beauty. " " Orchestra Great Need of Montreal », The Gazette, 11 avril 1930, 13. 
manifesté par les autorités politiques du Québec pour des projets d'éducation musicale s'adressant à un large public. Les institutions d'enseignement anglophones participent en grand nombre à la SAM, mais très peu d'écoles francophones les rejoignent. Ainsi, bien que la commission scolaire protestante ait nommé dès 1897 un responsable de l'enseignement du solfège et de la direction des chorales, ce n'est qu'en 1937, et après plusieurs revendications, que les commissions scolaires catholiques nommeront (et pour quelques années seulement) un directeur de l'enseignement du solfège dans leur secteur ${ }^{53}$.

Par ailleurs, l'initiative du DSC a ouvert la voie au dialogue dans le milieu musical. D'une part, cet événement annuel a été rehaussé par la participation de musiciens professionnels francophones à la direction artistique et au déroulement de certains concerts; d'autre part, le modèle de la SAM conduit à la création, en 1937, d'un nouvel organisme, le Festival de musique du Québec, qui assurera le relais en étendant ses activités à travers la province.

Devant l'indifférence de l'État à s'engager dans un tel projet communautaire, ces dames du DSC ont implanté à Montréal les modalités d'un partenariat entre le milieu des affaires et celui de la culture. Elles ont ainsi acquis un savoir-faire au sein de la gestion d'organismes culturels et ont contribué non seulement au développement de carrières spécifiques en ce domaine, mais également à la démocratisation de la culture musicale ${ }^{54}$.

Du côté francophone, on constate la quasi-absence de tradition de clubs féminins dans le milieu musical durant la période étudiée ${ }^{55}$. Qu'elle en est la raison? Pour contrer l'influence du Montreal Local Council of Women créé en 1893, la Fédération de la Société Saint-Jean-Baptiste a regroupé en 1907 les comités féminins existants en orientant leurs intérêts uniquement vers l'action sociale catholique. L'engagement culturel viendra plutôt d'initiatives individuelles de musiciennes et leur action sera destinée à la promotion de la jeune création canadienne contemporaine. En 1903, Céline Marier, professeure de chant réputée, organise un premier récital d'œuvres canadiennes en présence des membres de l'École littéraire de Montréal. D'autres suivront dans les années 1920 grâce aux initiatives d'Albertine Morin-Labrecque, de Jeanne Jarry et de $M^{\mathrm{me}} \mathrm{L}$. J. Tarte. Dans les années 1950, le succès des concerts annuels de la Société de musique canadienne reposera en grande partie sur le travail bénévole d'Isabelle Papineau-Couture. L'arrivée à Montréal en 1957 de l'animatrice Maryvonne Kendergi, dont la contribution à la promotion de la musique contemporaine est inestimable, ouvre un nouveau chapitre de l'histoire musicale du Québec.

53Seul le Conseil des arts et manufactures, sous l'égide de la Société Saint-Jean-Baptiste, offrait depuis le début du siècle ces cours au Monument national, sous la direction de J. J. Goulet et de J. B. Dubois.

54 Ces conclusions sont provisoires, car ce n'est que lorsque nous aurons cumulé suffisamment de renseignements sur l'ensemble des clubs musicaux féminins anglophones montréalais que nous pourrons avoir une vision plus juste de leur impact sur la vie culturelle du Québec.

$55 \mathrm{La}$ seule organisation dont on connaît l'existence, mais non les activités, est celle qu'Idola Saint-Jean, féministe de la première heure, fonde en 1908 : l'Association artistique des dames canadiennes. Voir La Patrie, 25 février 1911, 1. 


\title{
Résumé
}

L'étude présente d'abord le contexte dans lequel fut créé la Semaine de musique annuelle (1923-1937) à Montréal et propose une analyse du mode de fonctionnement, inspiré du modèle américain créé par Charles Milton Tremaine en 1920. L'auteure commente les données sur les collaborateurs, les commanditaires, les bourses, les lieux de concerts, les interprètes, le répertoire et les conférenciers, et fait ressortir les principaux éléments de la critique de ces événements. La conclusion met en évidence la dualité culturelle anglophone-francophone dans la conception de la musique et son rôle dans l'éducation, et souligne l'action des femmes dans la gestion d'organismes de diffusion de la musique au Québec.

\begin{abstract}
This study begins with an examination of the background against which was founded the annual Music-Week in Montreal (1923-1937), then presents an analysis of its system of operation, inspired by the American model created in 1920 by Charles Milton Tremaine. Information on the staff of the association, its sponsors, financial grants, concert venues, performers, repertory, and lecturers is discussed; the main points of the press reviews of the events are extracted. The anglophone-francophone cultural dualism with regard to the conception of music and of its role in education emerges clearly in the conclusion, which also highlights the active role of women in the running of organizations destined to spread music in Quebec.
\end{abstract}

